\title{
Automated Management of Quality Control System for Network Enterprise
}

\author{
J.P.T. Mo \\ CSIRO Manufacturing Science and Technology, Australia \\ Em:John.Mo@cmst.csiro.au
}

Keywords Quality Control System, Remote Operations Support, Web Technologies, Process Modelling

\begin{abstract}
One of the driving forces in manufacturing in the last decade is the demand of customers on the quality of the product they buy. However, as products become more complex and involve a lot of other subcontractors, interorganisational quality control functions require strong support from the organisations involved as well as an effective IT infrastructure. This paper describes a project involving a large transport equipment manufacturer using a methodology known as FIRST to develop an automated quality management process. The FIRST process provides a framework for the company to capture quality control information into a process model which can then be converted to the final user-end system automatically.
\end{abstract}

\section{INTRODUCTION}

It is generally accepted that quality is a keyword for winning current global competition in every business. Total Quality Management is one of prominent management strategies to win this competition [1]. In a company, the way to manage quality is by establishing a quality system. This comprises a systematic approach to plan, to do, to monitor and to control company's operations in achieving customer satisfaction [2].

In aviation industries, requirements for quality system are common practices. They are usually tied with safety requirements such as the airworthiness issues. Therefore, an aircraft manufacturing company must establish a quality system in its operation. Both quality manual and procedures must be in place. 
Along with internal efforts on quality achievement, a company needs to acquire quality materials, parts, or components from its suppliers. This is crucial because inconsistent quality of incoming resources may cause difficulties in achieving quality products.

There is an obvious conflict in buyer-supplier relationships. Suppliers may perceive that high quality products will increase manufacturing cost. On the contrary, buyers will always try to find a lower price for every material they purchase. The most common approach is to establish a supplier development program.

With the above imperatives, this project is motivated by the need of an aircraft manufacturing company to obtain such commitment on quality from its widely distributed suppliers.

\section{ROLES OF QUALITY SYSTEM DOCUMENTATION}

In quality management, there are two major roles of documentation. Firstly, system documentation demonstrates that the quality system is under control, clear and convincing. Management should know how the system is regulated. Employees need to comply with the system. Internal auditors should understand the system before they check the actual condition. Secondly, documentation on quality records is of authentic significance. To evaluate achievement level of quality parameters, records are needed. The evaluation process can then be used to determine corrective actions for process capability and product quality improvements.

A typical quality system in a company comprises a hierarchy of documents. This hierarchy forms a pyramid structure, which places quality policy at the top of the pyramid (Figure 1). Other quality documents must be developed based on the top level policy.

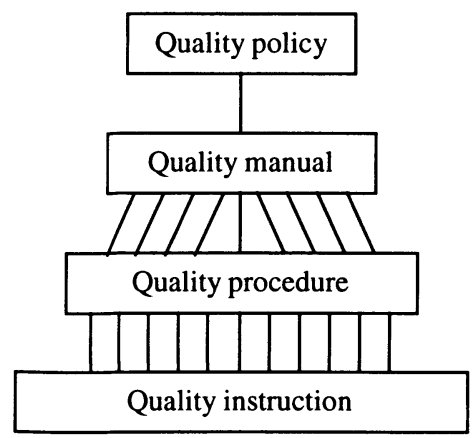

Figure 1 Hierarchy of Quality System Document 
Like other documentation systems, quality system documentation traditionally uses paper as its media. Up to now, it is still a popular media that keeps the consistency of information in a reasonable period of time.

There are several alternatives of documentation media, for example microfilms and optical disks. These media have considerably reduced the use of paper. However, for daily operation, they need high cost equipment, materials and processes. Moreover, by using these media, the documentation control system remains the same and it is difficult to propagate changes to lower levels.

Nowadays, efforts to reduce paper in human activities is growing. Companies may gain potential advantages of using a paperless documentation, especially in quality system. Several efforts have been started since the wide use of computer, but the developments are still in the initial stage [3].

\section{WEB-BASED QUALITY SYSTEM}

The use of computer is an important consideration in achieving paperless documentation system. The latest research has involved the Internet and Intranet technologies. These technologies promise a great benefit in distribution problems, because they will eliminate delays in delivery even though the users are located in a long distance.

A quality recording system may as well take advantage of web-based technologies by using capabilities of Java programming in its pages, thus making the system truely interactive and uniform. This makes it easier to enter and retrieve data.

An interesting result that comes out is when the recording system is combined with the web-based documentation. With an interactive interface, a seamless and consistent look-and-feel quality system may be produced. Last but not least, companies can benefit from the independence of web system on the users' platform. The investment for new computing systems is minimised.

\section{FIRST METHODOLOGY}

FIRST stands for Framework for Integration of Remote Support Technologies developed by CSIRO Manufacturing Science and Technology. FIRST methodology was initially developed to provide automatic creation of remote operation support system [4]. The main concept of the framework is to integrate the World Wide Web technology, hypertext markup language (HTML), interactive forms, multimedia, artificial intelligent, and supported 
by a knowledge based system. Besides easing remote customer support, the framework may benefit in reducing customer support related costs and minimise service delay time when used over long distances.

An important aspect of the framework is the use of an integrated knowledge-based system [5]. To enable this function, the system must understand the entire customer support process including the capability to collect facts and to navigate customers within the process. In traditional approaches, off line support is provided by experts performing such tasks in a direct mode.

In the FIRST methodology, this function is carried out using a knowledge repository, which is built through IDEF3 process modelling [6]. IDEF3 process modelling has capabilities to capture processes including logical and temporal relationships between activities in the processes. A fundamental treatment of IDEF3 to drive knowledge representation has been written by Mo and Menzel [7]. From users' view, operational cost for accessing remote system is quite low, because it uses web browsers, which is a free software.

\section{REMOTE QUALITY SYSTEM IMPLEMENTATION}

Using the same framework, a remote quality system capturing the quality system manuals, procedures and work instructions can be developed. Depending on the system design, additional scripts to access quality control database can also be attached to the remote support system.

The importance of knowledge repository is not only for initial publication of the quality system documents, but also for modifications that may apply during their life cycle. When change is required, the model is modified and the web-based support system is regenerated. Thus, this approach accommodates the documentation control function.

As stated before, the object of this project is to develop a candidate quality system for suppliers of an aircraft manufacturing company. Currently, this company has implemented a similar system with the same content, but in non-electronic media.

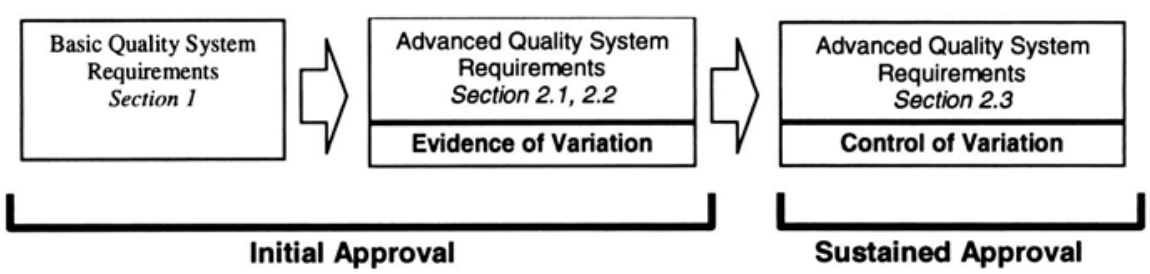

Figure 2 Quality System Approval Requirements 
To make the system easier to understand, an overall Quality System workflow is provided by the company (Figure 2). It illustrates the step-by step process and documentation requirements.

The project started with the creation of a system model. The system is divided into four different subsystem and function (Table 1).

Table 1 : Main Subsystems and Their Function

\begin{tabular}{ll}
\hline Subsystem & Functions \\
\hline AQS Concepts & $\begin{array}{l}\text { Overview and basic understanding of the newly introduced } \\
\text { system }\end{array}$ \\
\hline Basic Quality & $\begin{array}{l}\text { Descriptions of basic quality requirements that has been in } \\
\text { place }\end{array}$ \\
\hline Advanced Quality & $\begin{array}{l}\text { Description of new quality requirements for suppliers, } \\
\text { including recommended actions and flows to be accomplished. }\end{array}$ \\
Requirements & $\begin{array}{l}\text { Explanation of various tools those can be utilised in fulfilling } \\
\text { AQS Tools }\end{array}$ \\
& AQS requirements. \\
\hline
\end{tabular}

This documentation allows the main branch of the system model to be created as illustrated in Figure 3 and guides the users to select one of different subsystems they are interested to explore. A new user will easily decide to explore the Quality System Concept, while an expert will directly explore the Quality System Requirements to get the information he/she wants. Similarly, executives who will decide to implement the system can easily read the summary in the Quality System Concept section, while their technical staffs or subordinates will explore the details in the requirement sections.

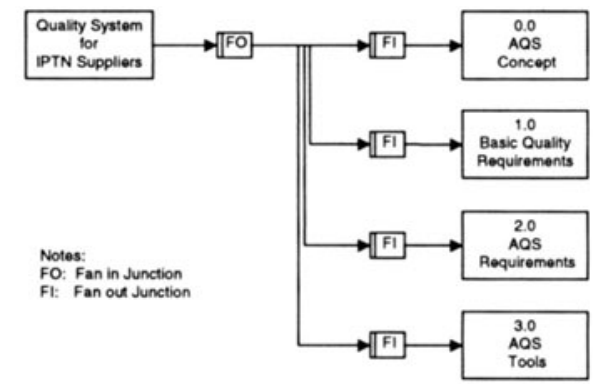

Figure 3 : Main Branch in IDEF3 Model

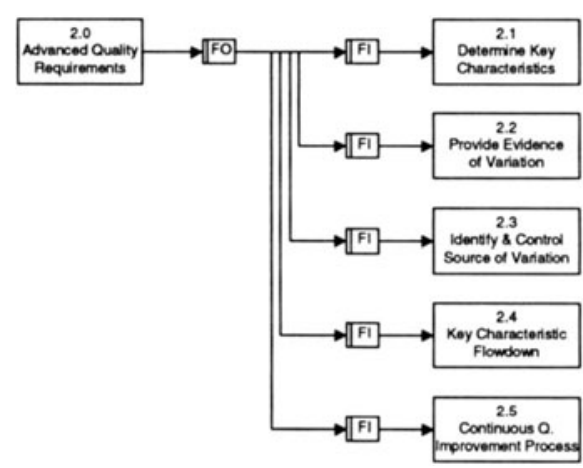

Figure 4 : Core Workflow Main Branch

The AQS (Advance Quality System) Requirements section is the core message in this documentation. So, the system is developed intensively in this section. The main objective is to guide the user to explore the 
requirements in a sequential basis. The users are guided to follow the information interactively with the system. After exploring a certain process, users will be linked to some possible pages that relate to that process. There are two kinds of links, viz. links to the next process that follows the current process and links to referred pages that contain related material and tools. With this arrangement, it is expected that users can be easily and systematically navigated through the system (Figure 4).

In the complete model, apart from the quality related processes, one can also see two additional processes attached to the model (Figure 5). They are Welcome and Thank You processes. The function of Welcome process is not only to give a welcome speech to users, but also is the starting process providing a fan-in junction leading to one of the next level of processes. This is important, because the next process after the Thank You process is a Home base of the model.

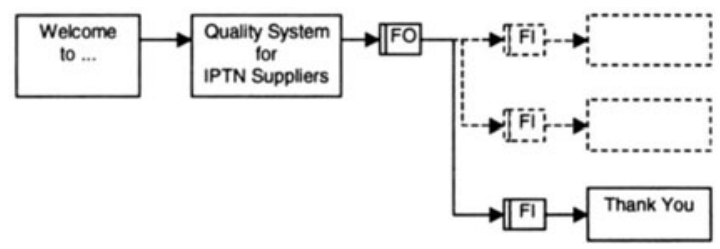

Figure 5 : Additional Processes

FIRST generates 101 web pages from the model. These pages build a web site and link one another providing trials of the quality system information and procedures. The FIRST methodology produces a start page in every site it develops. This page is always named as start.html. It represents the first process from which the model starts. For this project, the first process is Welcome page (Figure 6). A single click in the Next Page link word will start surfing the site.

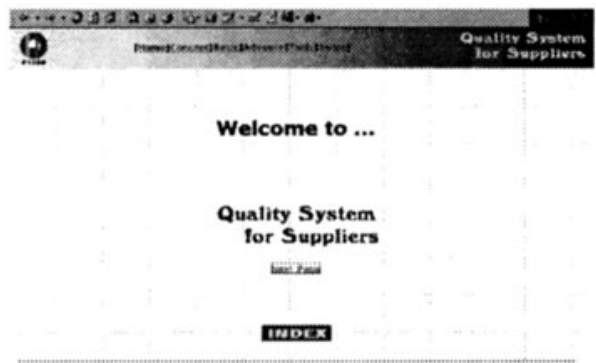

Figure 6: Welcome Page

It is impractical to explain the model step by step. However, to give an overall picture of how the model works, one can find four typical patterns of 
relationship among the processes in the model. They are simple continue pattern, yes - no pattern, multiple choice pattern, and reference pattern.

A simple-continue pattern is created in the model when the current process being modeled only have one possible link to the next one. Sometimes, this pattern can be used when we need to divide a long process into two or more process (Figure 6).

A yes-no pattern is needed when users are required to answer a yes-no question after evaluating the current process. If the user can identify the special cause of variation, then he/she will choose a 'yes' answer. This will cause the system to guide him/her to remove that special cause of variation, otherwise, the system will ask whether gauge variation study has been performed or not. For the example given in Figure 7, the site will bring the users to section 2.3.4 if they answer 'Yes' and to section 2.3.6 if they answer 'No'.

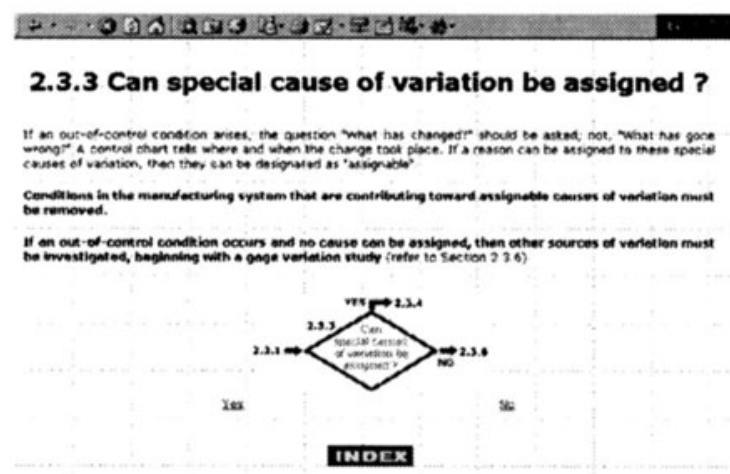

Figure 7 Yes-No Pattern Results

A multiple-choice pattern is created when users are advised to choose more than one possible answer. It is almost like yes-no pattern, but the answers to the question are more than simple yes or no. FIRST methodology has enabled this possibility by expressing all possible links in the current process/page. The link names are the possible page titles or ones those have been defined in 'process facts' when modelling. An example of the result is shown in Figure 8.

\section{CONCLUSION}

Sharing information over long distance is a potential benefit of the emergence of the internet/intranet. To exploit this benefit, CSIRO has developed a methodology called FIRST. This methodology enables systems for remote customer support through the World Wide Web to be created with an integrated process model driven knowledge based system. 
This project has implemented a Quality System using FIRST methodology. The main users of this system are suppliers of an aircraft manufacturing company to enable easy quality system access and maintenance. It is predicted that many areas in quality system may likely take advantages of this development, for example, supplier control, quality audit or even manufacturing control like measurement equipment control, facility and personnel certification and non-conformance material control.

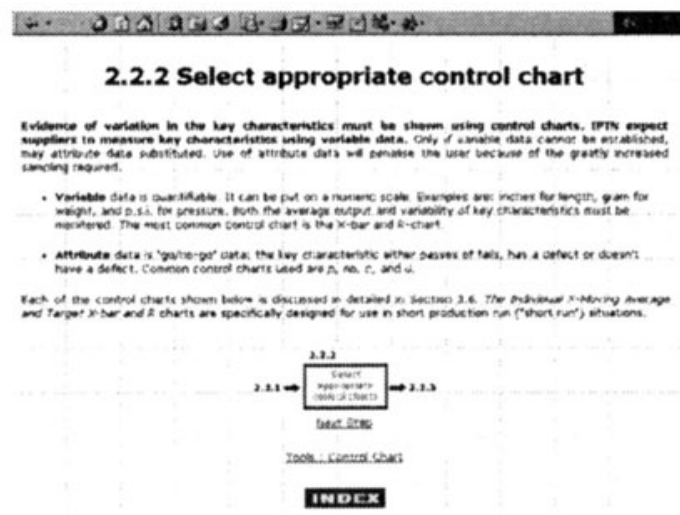

Figure 8: Multiple-Choice Pattern Results

\section{REFERENCES}

[1] Mo J.P.T., Chan A.M.S. (1997). "Strategy for the Successful Implementation of ISO 9000 for Small and Medium Manufacturers”, TQM Magazine, Vol.9, No.2, pp.135-145

[2] Scholtes, P.R. (1991). "The Team Handbook", pub. Joiner

[3] Koshy T.T., Gramopadhye A.K., Kennedy W.J., Ramu N.V. (1996) "Application of Hypertext Technology to Assist Maintenance on the Shop Floor", Computers and Industrial Engineering, Vol.30, No.2, pp.283-295

[4] CSIRO Manufacturing Science and Technology (1998). "FIRST-Framework for Integration of Remote Support Technologies”, Development Manual, Ver.1.1, January

[5] Mo, J.P.T, and Nemes, L. (1997). “A Knowledge Based Remote Diagnostics System", IFAC Symposium on Artificial Intelligence in Real Time Control, 22-25 September, Kuala Lumpur, Malaysia, pp.541-546

[6] Mo J.P.T., Nemes L. (1998). "Framework for automatic creation of remote operations support systems", Third International Conference on the Design of Information Infrastructure Systems for Manufacturing (DIISM '98), May 18-20, Fort Worth, Texas, U.S.A., pp.93-104

[7] Mo, J.P.T, Menzel, C. (1998). “An Integrated Process Model Knowledge Based System for Remote Customer Support”, Computers in Industry, Vol. 37, pp171-183. 